\title{
DNA methylation pattern in human zygotes and developing embryos
}

\author{
Helena Fulka ${ }^{1}$, Milan Mrazek ${ }^{2}$, Olga Tepla ${ }^{2}$ and Josef Fulka Jr ${ }^{1,2}$ \\ ${ }^{1}$ Center for Cell Therapy and Tissue Repair, VUZV Labs., Prague 10, Czech Republic and ${ }^{2}$ ISCARE IVF, Prague 9, \\ Czech Republic
}

Correspondence should be addressed to J Fulka Jr; Email: fulka.josef@vuzv.cz

\begin{abstract}
We report on observations of the global methylation/demethylation pattern of both pronuclei in human zygotes and in early embryos up to the blastocyst stage. Our results demonstrate that in about half of the zygotes examined the paternal chromatin was less methylated than the maternal chromatin. In the other half, both pronuclei exhibited the same intensity of labeling. The nuclei in developing embryos were intensively labeled for up to the four-cell stage; thereafter, a decline of labeling intensity was detected. Remethylation in some nuclei starts in late morulae. Surprisingly, and unlike the mouse, at the blastocyst stage the inner cell mass showed a weaker intensity of labeling than the trophectodermal cells.

Reproduction (2004) 128 703-708
\end{abstract}

\section{Introduction}

Normal embryonic development in mammals requires differential imprinting of both male and female genomes (Reik \& Walter 2001, Li 2002, Santos \& Dean 2004). After the oocyte is activated by sperm and completes meiosis, haploid oocyte chromosomes transform into a maternal pronucleus and the haploid sperm nucleus transforms into a paternal pronucleus (Yanagimachi 2003). When sperm chromatin protamines are replaced with oocyte cytoplasmic histones, the extensive demethylation of paternal, but not maternal, DNA occurs (Mayer et al. 2000, Oswald et al. 2000). This demethylation pattern was detected, after labeling with an antibody to 5-methylcytosine (anti-5-MeC), in all mammals tested so far (mouse, pig, rat and cattle), with the exception of sheep and rabbit (Rougier et al. 1998, Bourc'his et al. 2001, Dean et al. 2001, Wilmut et al. 2002, Shi et al. 2004). It has been further shown in mouse and bovine embryos that the methylation of DNA declines up to the eight-cell stage. In the mouse, the process of demethylation continues up to the morula stage (Dean et al. 2001, Santos et al. 2002). In blastocysts, however, evident differences were detected. In the mouse, the trophectoderm was undermethylated and the inner cell mass (ICM) was intensively methylated. On the other hand, in bovine blastocysts, both ICM and trophectoderm were methylated. This may indicate some species-specific differences. Logically, the most attractive subject would be the analysis of the methylation/demethylation pattern in human embryos.
In our study, the anti-5-MeC staining pattern has been examined in spare human embryos; that is, from zygotes up to the blastocyst stage.

\section{Materials and Methods}

Human zygotes were produced by conventional in vitro fertilization (IVF) or after ICSI (intracytoplasmic sperm injection) of oocytes that were not fully matured when aspirated from patient follicles. These oocytes completed maturation within a few hours when cultured in vitro. We have used these zygotes only when we obtained a reasonable number of high-quality zygotes from mature oocytes that were obtained from the same ovarian aspiration and patients did not consent to embryo freezing. The use of human zygotes for the evaluation of the demethylation pattern has been permitted by an internal IVF center scientific board (ISCARE) with the written consent of all patients.

Mouse zygotes were obtained from ICR mice previously stimulated with equine chorionic gonadotrophin (eCG; 5 IU) and injected with human CG (hCG; 5 IU) $40 \mathrm{~h}$ later. The animals were kept under standard conditions $\left(22^{\circ} \mathrm{C}, 60 \%\right.$ relative humidity, lights on 0700-1900 h). Females were then caged with ICR males and checked for the vaginal plug next morning. Then zygotes were isolated from oviducts and kept in culture until used for labeling. Eventually, they were cultured in KSOM (Specialty Media, Phillipsburg, NJ, USA) to reach the appropriate stage (blastocysts).

Both human and mouse zygotes were fixed at the expected G2-phase of the first cell cycle (mouse, 15-17 h 
after fertilization; human, $16-18 \mathrm{~h}$ after IVF or ICSI). Before fixation, zonae pellucidae were removed from zygotes or developing embryos by pronase treatment $(0.5 \%$ in PBS), and then the embryos were fixed in paraformaldehyde (4\% in PBS) at room temperature (mouse, $15 \mathrm{~min}$; human, $45 \mathrm{~min}$ ). The labeling with antimethylcytosine antibody (Eurogentec, Seraing, Belgium) was done basically as described by Santos et al. (2002). After fixation, embryos were washed in PBS and permeabilized with $0.2 \%$ Triton $X-100$ in PBS for 45 min at room temperature; then they were again washed in PBS and treated with $2 \mathrm{M} \mathrm{HCl}$ at room temperature for $30 \mathrm{~min}$. After washing in PBS, embryos were blocked with 5\% normal goat serum (Santa Cruz Biotechnology, Santa Cruz, CA, USA) in $0.2 \%$ Triton $\mathrm{X}-100$ in PBS at $37^{\circ} \mathrm{C}$ for $2 \mathrm{~h}$ and then incubated with anti-5-MeC antibody appropriately diluted in the blocking solution at $4{ }^{\circ} \mathrm{C}$ overnight. The primary antibody was detected by a goat antimouse secondary antibody coupled with FITC (Santa Cruz Biotechnology). The samples were stained by propidium iodide $(50 \mu \mathrm{g} / 0.5 \mathrm{ml})$. Then they were washed in PBS, mounted in Dabco antifade and inspected under Olympus IX71 fluorescent microscope (FITC or rhodamine filter).

The evaluation of total fluorescence intensity in relation to the nuclear size and processing of individual images was performed with AnalySIS software (Soft Imaging System GmbH, Münster, Germany), basically as described by Kim et al. (2002). The correction for the background was carried out before analyzing the fluorescent signal. The size of individual nuclei was measured by DNA staining with propidium iodide. For the quantification of 5-methylcytosine fluorescence intensity, the value from two-cell-stage embryos was arbitrarily set as $100 \%$, and the fluorescence intensity of each sample was compared to it. The numbers of analyzed cells for different stages were as follows: zygotes, 59; two-cell stage, 12; four-cell stage, 24; eight-cell stage, 30; morula, 35; early blastocyst, 41; late blastocyst, 45 - with reference, in each case, to the ICM and the trophectoderm.

The statistical analysis was performed with Statistica software (StatSoft, Prague, Czech Republic). Student's $t$-test was used to compare the values of relative fluorescence in different stage embryos.

Unless stated otherwise, all chemicals were purchased from Sigma (Prague, Czech Republic).

\section{Results}

In our experiments, all zygotes were fixed shortly before the onset of the first cleavage (from one- to two-cell stage) and then labeled with an antibody to 5-methylcytosine (5-MeC). It must also be noted that embryos came from different patients, and for every stage the labeling was done in at least three independent experiments. In total, we evaluated 59 morphologically high-quality; one-cell stage embryos that contained two centrally located and closely apposed pronuclei and the second polar body (Fig. 1A). As controls, late one-cell-stage mouse zygotes were used.

Although the paternal pronucleus in the mouse can be easily distinguished from the maternal pronucleus by its larger size, both pronuclei in normal human zygotes are the same size. To test the specificity of this method, we first labeled 72 one-cell-stage mouse embryos collected approximately $15-17 \mathrm{~h}$ after fertilization. In 66 of them, only the smaller pronucleus (maternal) was labeled, while the larger (paternal) pronucleus showed no or very weak labeling (Fig. 1B). Either the pronuclei in the remaining zygotes were without labeling (4), or both pronuclei showed the same intensity of labeling (2). In general, these observations confirmed the results published by others and also showed the convenience of our methodological approach.

Surprisingly, when human zygotes were labeled, the results differed dramatically from those obtained in the mouse. Of 59 zygotes evaluated, only 30 (51\%) showed the expected pattern where just one pronucleus was intensively labeled while the other one showed an evidently lower intensity of labeling (Fig. 1C). The intensity of labeling in the pronucleus with a lower fluorescence was about
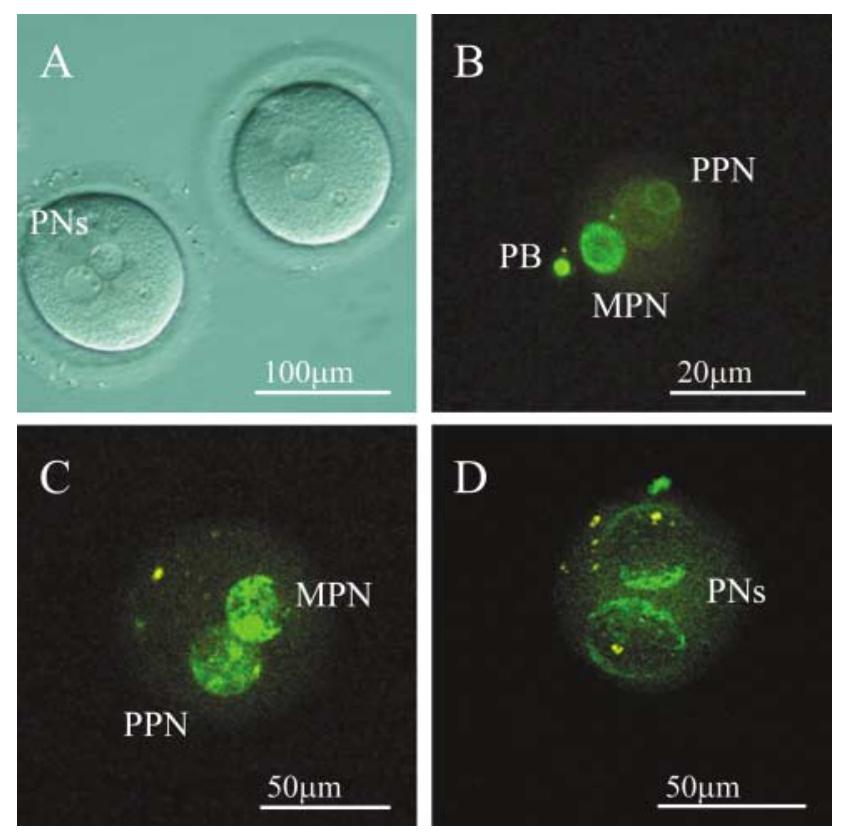

Figure 1 (A) Human one-cell-stage embryos with two closely apposed pronuclei (PNs). (B) The typical demethylation pattern in the onecell-stage mouse embryo. Only the maternal pronucleus (MPN) shows intensive labeling with an antimethylcytosine antibody, while the large paternal pronucleus (PPN) is without labeling. The second polar body (PB) is also labeled. (C) In about half of the human zygotes, one pronucleus (paternal - PPN) was less labeled, while the other one showed much higher intensity of labeling (maternal MPN). In human zygotes, both pronuclei are of the same size. (D) In the second half of the evaluated human zygotes, both pronuclei (PNs) showed the same intensity of labeling. 
$30 \%$ less than in the partner pronucleus. This difference was statistically significant $(P<0.05)$. In comparison with the paternal mouse, we have never observed that the pronucleus showed almost no labeling. We assumed that the pronucleus with a weaker labeling pattern was the paternal pronucleus. This assumption was confirmed when we evaluated some triploid dispermic zygotes with three pronuclei (two paternal and one maternal) and the second polar body. In this case, two pronuclei were weakly labeled while the remaining pronucleus showed a stronger intensity of labeling (not shown). Moreover, second polar bodies (maternal chromatin) always showed an intensive pattern of labeling. Interestingly, in the remaining morphologically high quality zygotes (29; $49 \%$ ), both pronuclei showed roughly the same intensity of labeling (Fig. 1D). In contrast to the mouse, we have never observed pronuclei without any labeling.

Next, we examined the pattern and quantified the fluorescence intensity of methylation in spare human embryos in two-cell, four-cell, eight-cell, morula and blastocyst stages. For every stage, we examined at least 10 morphologically high-quality embryos. In two- and four-cell-stage embryos, all blastomeres were intensively labeled (Fig. 2A). The evident drop of labeling intensity was detected in the next cleavage stage embryos; that is, in the eight-cell stage (Fig. 2B). The same weak pattern was seen
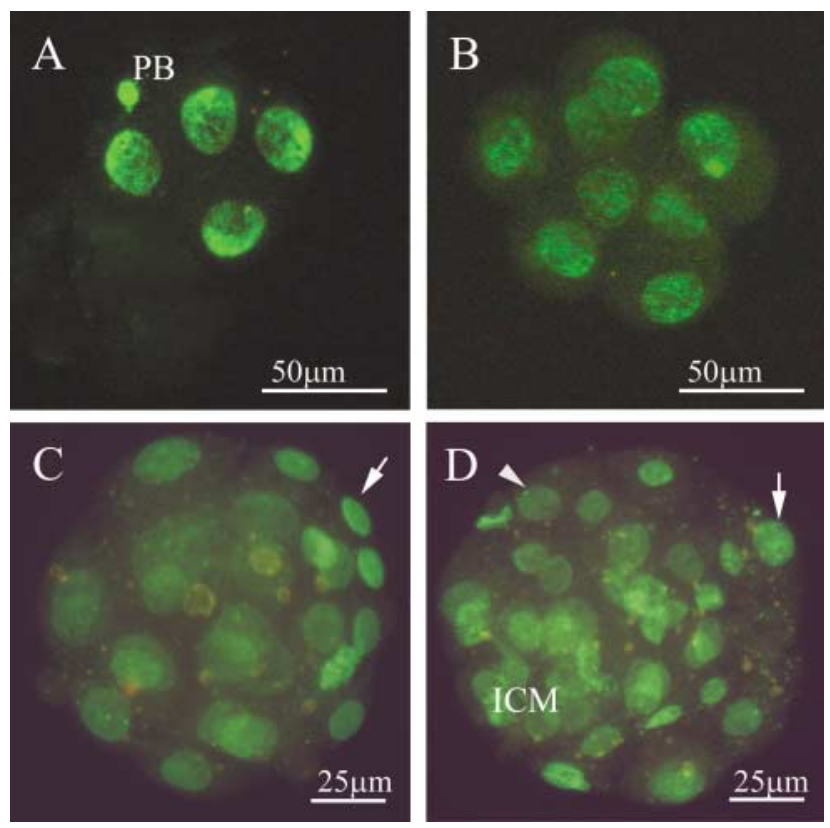

Figure 2 (A) Four-cell-stage human embryo with heavily labeled nuclei in each blastomere. PB: polar body. (B) Eight-cell-stage embryonic nuclei showing the evident decrease of 5-MeC labeling intensity. (C) The weak intensity of 5-MeC labeling is evident for almost all morula-stage nuclei, but in some of them (arrow) the intensity of labeling is evidently higher. (D) The weak intensity of labeling is typical also of the early blastocyst stage (arrowhead). As in the morula, some nuclei show a very intensive methylation pattern (arrow). ICM: inner cell mass. in the early morula stage (Fig. 2C); however, as development advanced (early blastocysts), some nuclei were more intensively labeled while in others the weak intensity of labeling persisted (Fig. 2D). The most surprising results were obtained after the labeling of fully expanded blastocysts (Fig. 3A). We expected a pattern of labeling similar to what is typical of the mouse; that is, heavily labeled ICM and no labeling in the trophectoderm. This pattern was also observed in our control mouse blastocysts (Fig. 3B). However, in human embryos, a completely different pattern of labeling was consistently detected. Here, trophectodermal (TE) cells were very intensively labeled while the ICM showed a weaker intensity of labeling (Fig. 3C). It must be noted that only blastocysts perfect in appearance were used (Fig. 3A and D). The measurement of the nuclear size and fluorescence intensity in late blastocysts revealed that the size of nuclei between TE and ICM cells did not differ significantly $(P>0.05)$; however, a significant difference was detected in the relative intensity of fluorescence $(P<0.05)$. The quantification of fluorescence intensity is presented in Fig. 4. To test whether these results were caused by a restricted penetration of the anti-5-methylcytosine antibody, BrdU labeling was performed as control. No differences in the fluorescence signal intensity were observed between the ICM and TE cells.
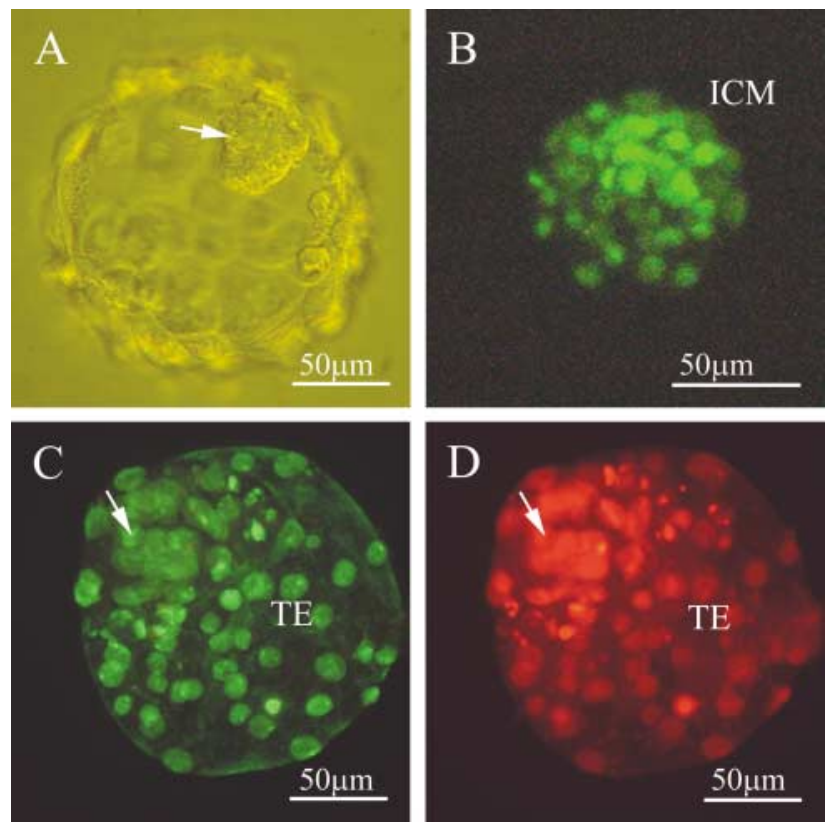

Figure 3 (A) An example of a blastocyst used for 5-MeC labeling. Note the prominent ICM (arrow). (B) Mouse blastocyst with heavily methylated inner cell mass (ICM) and trophectoderm that is evidently undermethylated. (C) In the fully expanded human blastocyst, the ICM (arrow) cell nuclei are moderately methylated while the trophectodermal (TE) cells exhibit an evidently more intensive pattern of labeling. (D) The blastocyst shown in Fig. 3C contains more than 100 cells with a distinct ICM (arrow). 


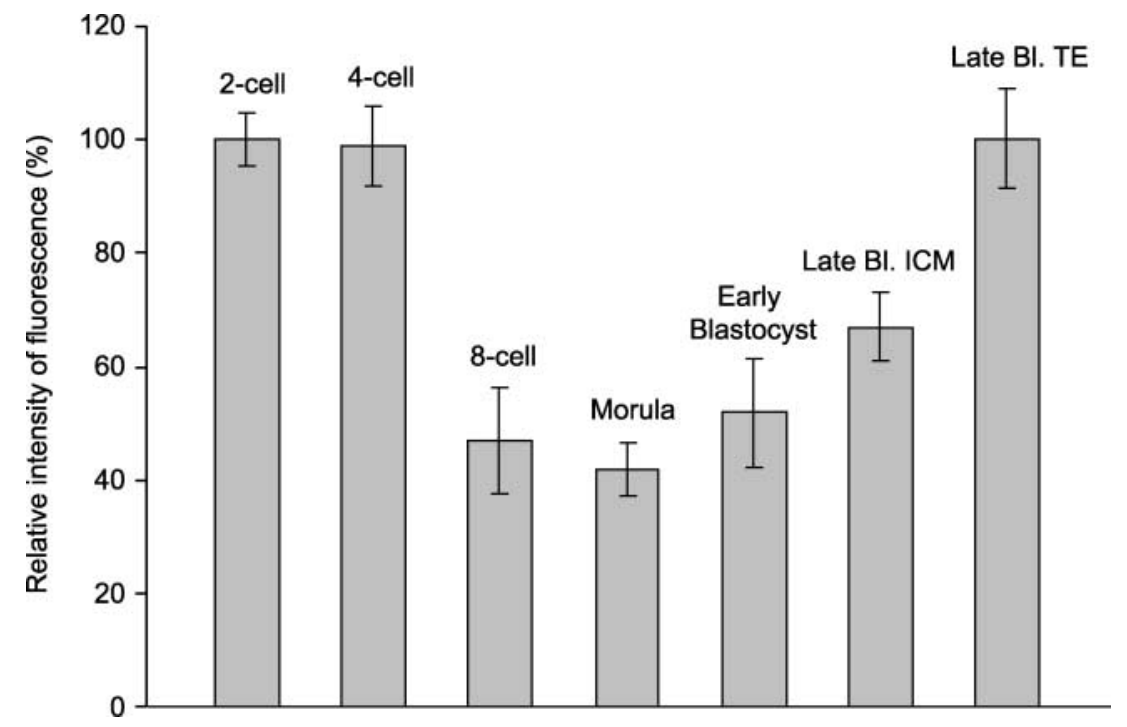

Figure 4 Changes in the methylation labeling intensity in human embryos. Each column represents the relative intensity of labeling against 5 -methylcytosine in relation to the nuclear size for different embryonic stages. For the blastocyst stage, the ICM cells and TE cells were analyzed separately. The intensity of fluorescence of the two-cell stage was set as $100 \%$ (error bars \pm S.E.M.). The relative fluorescence intensities were compared between different stages and between TE cells and the ICM. The relative intensity significantly decreased between the four- and eight-cell stages $(P<0.001)$ and rose again between the morula and late blastocyst (BI.) stages $(P<0.001)$.

\section{Discussion}

In general, our results show that, as in other mammals tested so far (mouse, pig, rat and cattle), also in human zygotes the paternal chromatin undergoes active demethylation soon after fertilization. This has been also confirmed recently by Beaujean et al. (2004a), but, in contrast to our results, these authors observed demethylation in all human zygotes (eight) examined. It has been shown in the mouse that the process of active demethylation starts even before the onset of the S-phase (Santos et al. 2002). For obvious ethical reasons, it is impossible to test whether the same applies to human zygotes.

It has been shown recently that the demethylation process is facilitated by the oocyte cytoplasm, as well as by a sperm-derived factor and by male chromatin composition. Interestingly, sheep sperm DNA can be demethylated in mouse oocytes, and mouse sperm DNA is demethylated in sheep oocytes (Beaujean et al. 2004b). Only in the sheep and rabbit do both pronuclei remain to be highly methylated. Interestingly, in their recent review article, Santos and Dean (2004) mentioned that they had also observed limited demethylation of the paternal chromatin in sheep zygotes. This may indicate that the influence of some subtle technical aspects cannot be ruled out. In general, it seems that human, as well as bovine, zygotes represent an intermediate stage between the mouse and the sheep, as we have never observed paternal pronuclei that were completely without labeling. The decline of labeling can be detected in the mouse from the two-cell embryonic stage to the morula. Then, de novo methylation can be detected in the blastocyst. In this species, the ICM is intensively methylated while the TE cells show no signs of methylation. The situation in bovine embryos differs; the gradual demethylation continues up to the eight-cell stage. Thereafter, de novo methylation begins. Interestingly, in the blastocyst, the ICM contains highly methylated nuclei while the TE cells are only moderately methylated. We observed a completely different situation in human embryos, where the ICM showed a weak (moderate) pattern and TE cells were heavily methylated. These distinct methylation patterns may reflect further differences occurring much later: that is, the process of implantation and placenta formation (Malassine et al. 2003). The expression of certain cell-surface antigens differs also during the early embryonic development between mouse and human preimplantation-stage embryos (Henderson et al. 2002). It also seems to us that the onset of the demethylation processes can be associated with the major burst of embryonic genome activation that occurs in the mouse at the two-cell stage, in human embryos at the four-cell stage and in bovine embryos at the eight-cell stage (Tesarik et al. 1988, Kopecny et al. 1989, Schultz 2002).

In general, DNA methylation is associated with gene silencing and the formation of heterochromatin. Both male and female gametes are highly specialized and are, in fact, terminally differentiated cells. The very early embryo is constituted from totipotent blastomeres; thus, it is not surprising that an extensive reprogramming of parental genomes must occur. It has also been postulated that, with continuing differentiation, the cell first gains a lineage and later a tissue-specific pattern of methylation that is linked to a specific pattern of gene expression (Reik et al. 2003). This covalent modification of DNA seldom acts alone. It is involved in changing the chromatin structures to repress gene expression (Meehan 2002, Grewal \& Moazed 2003) through a wide range of methyl-CpG binding domain (MBD) proteins, such as MeCP2, which can interact with different histone-modifying enzymes for example histone deacetylases (HDACs). In the mammalian embryo, the genome must be activated very soon after fertilization. The observed demethylation might therefore be linked to this step, as well as to resetting of the genetic program inherited from previous generations. 
The high incidence (nearly half) of zygotes with similarly labeled pronuclei is surprising. Typically, only $10 \%$ of mouse zygotes show an aberrant pattern of labeling (Barton et al. 2001). The possibility that our experiments used zygotes where the demethylation had just begun can be ruled out. All evaluated zygotes exhibited closely apposed pronuclei of the same size. Moreover, under our conditions, the cleavage of zygotes produced in the same way occurs within the next $2-4 \mathrm{~h}$. We must, however, realize that embryos used for labeling came from patients with different forms of fertility problems. It is possible that the percentage of normal demethylation pattern will be higher in an average human population.

It has been recently demonstrated by Santos et al. (2003) and Beaujean et al. (2004c), in cloned bovine and sheep embryos, that the frequency of the normal/abnormal demethylation pattern closely reflects the developmental ability of reconstructed embryos. Clearly, for ethical reasons, the human material necessary for precise and detailed evaluation will always be limited. We believe that our data convincingly demonstrate that in humans a different pattern of methylation/demethylation exists during early embryonic development.

It has become clear that defects in genomic imprinting and in other epigenetic processes result in aberrant development of the embryo and may have serious developmental consequences (Gosden et al. 2003, Kelly \& Trasler 2004, Lucifero et al. 2004). It remains, however, to be determined whether the extent of paternal chromatin demethylation in human zygotes is the cause of infertility or affects the development of embryos. We are convinced that detailed knowledge of the very early embryonic developmental processes is essential also for cell therapies in which spare embryos could be used as the source of human embryonic stem cells, as well as for 'therapeutic cloning' (Smith 2001, Magdinier et al. 2002, Geuns et al. 2003, Rhind et al. 2003).

\section{Acknowledgements}

J F Jr's laboratory gratefully acknowledges grants MSMT LN O0A 065 and GACR 524/02/0032 in support. H F thanks Anne McLaren for helpful discussion and comments on results.

\section{References}

Barton SC, Arney KL, Shi W, Niveleau A, Fundele R, Surani MA \& Haaf T 2001 Genome-wide methylation patterns in normal and uniparental early mouse embryos. Human Molecular Genetics 26 2983-2987.

Beaujean N, Hartshorne G, Cavilla J, Taylor J, Gardner J, Wilmut I, Meehan R \& Young L 2004a Non-conservation of mammalian preimplantation methylation dynamics. Current Biology $\mathbf{1 4}$ R266-R267.

Beaujean N, Taylor JE, McGarry M, Gardner JO, Wilmut I, Loi P, Ptak G, Galli C, Lazzari G, Bird A, Young LE \& Meehan RR 2004b The effect of interspecific oocytes on demethylation of sperm DNA. PNAS 101 7636-7640.
Beaujean N, Taylor J, Gardner J, Wilmut I, Meehan R \& Young L 2004c Effect of limited DNA methylation reprogramming in the normal sheep embryo on somatic cell nuclear transfer. Biology of Reproduction 71 185-193.

Bourc'his D, Le Bourhis D, Patin D, Niveleau A, Comizzoli P, Renard JP \& Viegas-Pequignot E 2001 Delayed and incomplete reprogramming of chromosome methylation patterns in bovine cloned embryos. Current Biology 11 1542-1546.

Dean W, Santos F, Stojkovic M, Zakhartchenko V, Walter J, Wolf E \& Reik W 2001 Conservation of methylation reprogramming in mammalian development: aberrant reprogramming in cloned embryos. PNAS 98 13734-13738.

Geuns E, De Rycke M, Van Steirteghem A \& Liebaers I 2003 Methylation imprints of the imprint control region of the SNRPN-gene in human gametes and preimplantation embryos. Human Molecular Genetics 12 2873-2879.

Gosden R, Trasler J, Lucifero D \& Faddy M 2003 Rare congenital disorders, imprinted genes, and assisted reproductive technology. Lancet 361 1975-1977.

Grewal SIS \& Moazed D 2003 Heterochromatin and epigenetic control of gene expression. Science 301 798-802.

Henderson JK, Draper JS, Baillie HS, Fishel S, Thomson JA, Moore H \& Andrews PW 2002 Preimplantation human embryos and embryonic stem cells show comparable expression of stagespecific embryonic antigens. Stem Cells 20 329-337.

Kelly TLJ \& Trasler JM 2004 Reproductive epigenetics. Clinical Genetics 65 247-260.

Kim JM, Ogura A, Nagata M \& Aoki F 2002 Analysis of the mechanisms for chromatin remodeling in embryos reconstructed by somatic nuclear transfer. Biology of Reproduction 67 760-766.

Kopecny V, Flechon JE, Camous S \& Fulka J Jr 1989 Nucleologenesis and the onset of transcription in the eight cell bovine embryo: fine structural autoradiographic study. Molecular Reproduction and Development 1 79-90.

Li E 2002 Chromatin modification and epigenetic reprogramming in mammalian development. Nature Reviews Genetics 3 $662-673$.

Lucifero D, Chaillet JR \& Trasler JM 2004 Potential significance of genomic imprinting defects for reproduction and assisted reproductive technology. Human Reproduction Update 10 3-18.

Magdinier F, Giscard d'Estaing S, Peinado C, Demirci B, Berthet C, Guerin JF \& Dante R 2002 Epigenetic marks at BRCA1 and p53 coding sequences in early human embryogenesis. Molecular Human Reproduction 8 630-635.

Malassine A, Frendo JL \& Evain-Brion D 2003 A comparison of placental development and endocrine functions between the human and mouse model. Human Reproduction Update 9 531-539.

Mayer W, Niveleau A, Walter J, Fundele R \& Haaf T 2000 Demethylation of the zygotic paternal genome. Nature 403 501-502.

Meehan RR 2002 DNA methylation in animal development. Seminars in Cell and Developmental Biology 14 53-65.

Oswald J, Engemann S, Lane N, Mayer W, Olek A, Fundele R, Dean W, Reik W \& Walter J 2000 Active demethylation of the paternal genome in the mouse zygote. Current Biology 10 475-478.

Reik W \& Walter J 2001 Genomic imprinting: parental influence on the genome. Nature Reviews Genetics 2 21-30.

Reik W, Santos F, Mitsuya K, Morgan H \& Dean W 2003 Epigenetic asymmetry in the mammalian zygote and early embryo: relationship to lineage commitment? Philosophical Transactions of the Royal Society of London. Series B: Biological Sciences 358 1403-1409.

Rhind SM, Taylor JE, de Sousa PA, King TJ, McGarry M \& Wilmut I 2003 Human cloning: can it be made safe? Nature Reviews Genetics 4 855-864.

Rougier N, Bourc'his D, Molina Gomes D, Niveleau A, Plachot M, Paldi A \& Viegas-Pequignot E 1998 Chromosome methylation pattern during mammalian preimplantation development. Genes and Development 12 2108-2113. 
Santos F \& Dean W 2004 Epigenetic reprogramming during early development in mammals. Reproduction 127 643-651.

Santos F, Hendrich B, Reik W \& Dean W 2002 Dynamic reprogramming in DNA methylation in the early mouse embryo. Developmental Biology 241 172-182.

Santos F, Zakhartchenko V, Stojkovic $M$, Peters A, Jenuwein $T$, Wolf E, Reik W \& Dean W 2003 Epigenetic marking correlates with developmental potential in cloned bovine preimplantation embryos. Current Biology 13 1116-1121.

Schultz RM 2002 The molecular foundations of the maternal to zygotic transitions in the preimplantation embryo. Human Reproduction Update 8 323-331.

Shi W, Dirim F, Wolf E, Zakhartchenko V \& Haaf T 2004 Methylation reprogramming and chromosomal aneuploidy in in vivo fertilized and cloned rabbit preimplantation embryos. Biology of Reproduction 71 340-347.

Smith AG 2001 Embryo-derived stem cells: of mice and men. Annual Reviews in Cell and Developmental Biology 17 435-462.
Tesarik J, Kopecny V, Plachot M \& Mandelbaum J 1988 Early morphological signs of embryonic genome expression in human preimplantation development as revealed by quantitative electron microscopy. Development 128 15-20.

Wilmut I, Beaujean N, de Sousa PA, Dinnyes A, King TJ, Paterson LA, Wells DN \& Young LE 2002 Somatic cell nuclear transfer. Nature 419 583-586.

Yanagimachi R 2003 Fertilization and development initiation in orthodox and unorthodox ways: from normal fertilization to cloning. Advances in Biophysics 37 49-89.

Received 27 February 2004

First decision 22 April 2004

Revised manuscript received 27 July 2004

Accepted 10 September 2004 